techniques can be used in a total knee replacement (TKR) surgery, by evaluating the patient's knee kinematic and contact forces. This would beneficent to optimise the geometry of the TKR device for the patient's knee.

\section{B-15 \\ Comparison of Osteoarthritic Knee Kinematics and Kinetics with Age Matched Healthy Individuals}

P. Worsley, M. Stokes, M. Taylor. University of Southampton, UK

It has long been established that individuals who suffer from osteoarthritis (OA) present with altered kinematics and kinetics during gait. Many studies have looked at the knee kinematics and kinetics, however the consequences of this asymmetry on the non-effected limb has not been explored. This study aims to investigate the knee kinematics and kinetics during gait for pre-operative OA patients when compared to healthy age and sex matched individuals.

Fifteen pre-operative (Knee Arthroplasty; KA) OA patients, and 10 age and sex matched healthy $(\mathrm{H})$ individuals were recruited, with institutional and hospital ethical approval sought prior to testing. Each participant underwent function screening tests using a 12 Item Oxford Knee Score and the Western Ontario and McMaster University Osteoarthritis Index (WOMAC). Participant's gait was then analysed in a motion capture session (VICON, Oxford, UK) during five trials within one session. Motion capture and force plate data were then processed into participant specific musculoskeletal models using AnyBody inverse dynamic modelling software (Aalborg, Denmark).

Results showed significantly lower perceived ability in activities of daily living in the OA group $(\mathrm{p}<0.001)$, with mean WOMAC and 12 item Oxford Knee scores of $50.2(\mathrm{H}=0)$ and $20.8(\mathrm{H}=48)$ respectively. Kinematics showed a large difference between groups in the range of motion of the knee, with the OA group exhibiting a average $6.2^{\circ}\left(\mathrm{H}=0.1^{\circ}\right)$ less peak extension and $3.9^{\circ}\left(\mathrm{H}=0.1^{\circ}\right)$ less peak flexion between affected and unaffected limbs during gait (Table 1). Knee kinetics showed considerable differences between groups, with increased valgus-varus and internal-external rotation moments (Table 1 ) in the OA group. The affected limb also exhibited decreased flexion moment $(2.7 \% \mathrm{BW} \times \mathrm{Nm})$ during stance phase of gait (Table 1)

Table 1. Peak mean Knee kinetics during $100 \%$ of the gait cycle.

\begin{tabular}{lllllllll}
\hline & \multicolumn{9}{l}{ Kinematics and Kinetics } \\
\cline { 2 - 9 } & $\begin{array}{l}\text { Flexion } \\
\text { (deg.) }\end{array}$ & $\begin{array}{l}\text { Extension } \\
\text { (deg.) }\end{array}$ & $\begin{array}{l}\text { Axial N } \\
\text { \%BW }\end{array}$ & $\begin{array}{l}\text { AP N } \\
\text { \%BW }\end{array}$ & $\begin{array}{l}\text { ML N } \\
\text { \%BW }\end{array}$ & $\begin{array}{l}\text { VV Nm } \\
\text { \%BW }\end{array}$ & $\begin{array}{l}\text { IE Nm } \\
\text { \%BW }\end{array}$ & $\begin{array}{l}\text { Flexion } \\
\text { moment } \\
\text { Nm \%BW }\end{array}$ \\
\hline OA affected & $59.4^{*}$ & $13.2^{*}$ & 308.6 & 58.6 & 8.1 & $12.1^{*}$ & $3.0^{*}$ & $2.7^{*}$ \\
& $(5)$ & $(6.9)$ & $(62.4)$ & $(35.8)$ & $(11.4)$ & $(6.1)$ & $(1.3)$ & $(2.5)$ \\
OA unaffected & 63.3 & 6.9 & 327.5 & 54.2 & 8.6 & $14.8^{*}$ & $3.4^{*}$ & 2.8 \\
& $(3.9)$ & $(6.3)$ & $(66.2)$ & $(33.4)$ & $(11.3)$ & $(9.7)$ & $(1.6)$ & $(2.2)$ \\
Healthy & 63.1 & 6.1 & 306.4 & 78.16 & $14.6^{*}$ & 6 & 0.7 & 3.9 \\
& $(2.1)$ & $(3.2)$ & $(37.1)$ & $(17.6)$ & $(12.3)$ & $(1.4)$ & $(0.4)$ & $(1.9)$ \\
\hline
\end{tabular}

Kinetics are normalised to \% of body weight (BW). Significant differences are highlighted with an asterix Anterior-posterior reaction (AP), mediolateral reaction (ML), valgus-varus torque (VV), internal-externa rotation torque (IE). Standard Deviations in brackets.

These findings show a marked reduction in perceived functional ability in pre-operative knee arthroplasty patients, which had direct effect on the symmetry of their gait. Kinematics and kinetics show significant differences between the healthy individuals and the $\mathrm{OA}$ group but also differences between the affected and unaffected limbs. Also, standard deviation of kinematics and kinetics in the $\mathrm{OA}$ group was substantially greater than that of the healthy. It has been shown that KA patients retain asymmetry in gait for years after the procedure, with evidence of $37 \%$ of primary KA patients receiving a replacement on the contra lateral limb within ten years. Perhaps the asymmetry, and associated altered loading, contributes to contralateral trauma and is clearly worthy of further exploration.

\section{B-16}

\section{Analysis of Knee Kinematics with Computer Navigation}

K. Deep, F. Picard, J. Baines, A.H. Deakin. Golden Jubilee National Hospital, UK

For the purpose of surgical correction during arthroplasty, arthritic knees are generally thought to be either varus (bow-legged) or valgus (knock-kneed). Soft tissue releases are done in accordance with this alignment as assessed by the clinical deformity and plain antero-posterior (AP) radiographs of the extended knee. However the used of computer aided navigation during total knee replacement allows the measurement of arthritic knee kinematics rather than just alignment in extension. We performed 283 total knee replacements with computer aided navigation. Two image-free navigation systems were used. Knee kinematics was recorded before and after the prosthesis implantation. This included measurement of the mechanical femoral tibial angle throughout the range of motion. The majority of knees did not behave in a consistent varus or valgus fashion. The deformities were classified into different groups depending on the behavior of the knee in coronal plane as it was moved from extension to flexion, with $2^{\circ}$ was taken as minimum deviation to signify change. The following classifications were used: Neutral as $1 \mathrm{~A}$, if deformity remains the same it is $1 \mathrm{~B}$. Increasing deformity is $2 \mathrm{~A}$ and decreasing deformity is described without reaching the neutral; 2B. Decreasing deformity reaches neutral described as 3. Decreasing deformity and crosses to opposite (varus to valgus and valgus to varus) deformity as $4 \mathrm{~A}$. When deformity first increases and then decreases but does not reach neutral it is $4 \mathrm{~B}$. If the deformity firstly increases and then decreases to neutral it is defined as $4 \mathrm{C}$. Deformity first increases and then decreases to cross over to opposite deformity. For classifications $1 \mathrm{~A}-4 \mathrm{C}$ knees were also divided based on whether they were varus or valgus in extension. Pre-operatively the commonest deformity was $2 \mathrm{~A}$ for varus knees and $4 \mathrm{~A}$ for valgus knees (Table 1 ). Group 1A, which corresponds to the traditional assessment of the deformity in extension being accurate, was only seen in $7 \%$ of knees (Table 1).

Table 1: Pre Operative numbers

\begin{tabular}{|c|c|c|c|c|c|c|}
\hline \multirow[t]{2}{*}{ Group } & \multicolumn{2}{|c|}{$\underline{\text { Neutral }}$} & \multicolumn{2}{|c|}{ Varus } & \multicolumn{2}{|c|}{ Valgus } \\
\hline & $\mathrm{n}$ & $\%$ & $\mathrm{n}$ & $\%$ & $\mathrm{n}$ & $\%$ \\
\hline Neutral & 1 & 0.4 & & & & \\
\hline $1 \mathrm{~A}$ & & & 16 & 5.7 & 3 & 1.1 \\
\hline 1B & & & 14 & 4.9 & 5 & 1.8 \\
\hline $2 \mathrm{~A}$ & & & 52 & 18.4 & 4 & 1.4 \\
\hline $2 B$ & & & 21 & 7.4 & 4 & 1.4 \\
\hline 3 & & & 43 & 15.2 & 17 & 6 \\
\hline $4 \mathrm{~A}$ & & & 36 & 12.7 & 25 & 8.8 \\
\hline $4 \mathrm{~B}$ & & & 11 & 3.9 & 7 & 2.5 \\
\hline $4 C$ & & & 9 & 3.2 & 13 & 4.6 \\
\hline
\end{tabular}

Traditional releases of medial or lateral structures without realising the true picture of what happens when the knee is flexed may not be correct. From this study it is clear that not all arthritic varus or valgus knees behave in the same way. Some of the releases performed conventionally may not be required or need to be modified depending on the knee kinematics. 